\title{
Observation of reconnection pulses by Cluster and Double Star
}

\author{
X. H. Deng ${ }^{1}$, R. X. Tang ${ }^{1}$, R. Nakamura ${ }^{2}$, W. Baumjohann ${ }^{2}$, T. L. Zhang ${ }^{2}$, P. W. Daly ${ }^{3}$, H. Rème ${ }^{4}$, C. M. Carr ${ }^{5}$, \\ A. Balogh ${ }^{5}$, Z. X. Liu ${ }^{6}$, and J. F. Wang ${ }^{1}$ \\ ${ }^{1}$ Institute of Electronics and Information, Wuhan University, Wuhan, Hubei, 430079, P. R. China \\ ${ }^{2}$ Space Research Institute, Austrian Academy of Sciences, 8042 Graz, Austria \\ ${ }^{3}$ Max Planck Institute for Solar System Research, 37191 Katlenburg-Lindau, Germany \\ ${ }^{4}$ Centre d'Etude Spatiale des Rayonnements, 31028 Toulouse, Cedex 4, France \\ ${ }^{5}$ Space and Atmospheric Phys. Group, Blackett Lab., Imperial College, London SW72BZ, UK \\ ${ }^{6}$ Center for Space Science and Applied Research, Chinese Academy of Sciences, Beijing 100080, P. R. of China
}

Received: 23 February 2005 - Revised: 14 July 2005 - Accepted: 19 August 2005 - Published: 8 November 2005

Part of Special Issue "Double Star - First Results"

\begin{abstract}
During a reconnection event on 7 August 2004, Cluster and Double Star (TC-1) were near the neutral sheet and simultaneously detected the signatures of the reconnection pulses. AT 22:59 UT tailward flow followed by earthward flow was detected by Cluster at about $15 R_{E}$, while earthward plasma flow followed by tailward flow was observed by TC- 1 at about $10 R_{E}$. During the flow reversal from tailward to earthward, the magnetic field $B_{z}$ changed sign from mainly negative values to positive, and the $X$ component of the magnetic curvature vector switched sign from the tailward direction to the earthward direction, which indicates that the reconnection site (X-line) moved tailward past the Cluster constellation. By using multi-point analysis and observation of energetic electron and ion flux, we study the movement and structure of the current sheet and discuss the braking effect of the earthward flow bursts in the inner magnetosphere.
\end{abstract}

Keywords. Space plasma physics (Magnetic reconnection) - Magnetospheric physics (Magnetotail; Storms and substorms)

\section{Introduction}

The temporal and spatial evolution of a magnetic substorm is an important and enduring problem in space plasma physics. The dynamics of the near-Earth plasma sheet has proven to be event more complex than those of the deep tail. Opinions on the physical processes which initiate the substorm onset in the magnetotail are still divergent. The near-Earth neutral line model (Baker et al., 1996) suggests that magnetic reconnection is the primary process causing the current disruption,

Correspondence to: X. H. Deng

(dengxh@public.wh.hb.cn) the formation of the current wedge and other consequences. The current disruption model (Lui et al., 1991) advocates that a key process is the disruption of the thin near-Earth current sheet, and magnetic reconnection is launched after some delay at a more distant location in the tail. To a large extent, the disagreement was due to the lack of direct and well-confined conjugate observations made on the ground, as well as in the near-tail and midtail. Coordinate multi-satellite and multiinstrument data analysis of individual events are most appropriate to establish the topology of a reconnection configuration and its causal relationship to substorm onset (Sergeev et al., 1996; Slavin et al., 2002). Its solution will entail a mature understanding of where and when these events begin, how and from where they draw their energy, and the manner in which this energy is transferred and dissipated throughout geospace.

Double Star is the first mission launched by China to explore the Earth's magnetosphere. The first of the two spacecraft (TC-1) was launched into an elliptical orbit on 29 December 2003 and the second spacecraft (TC-2) was launched into a polar orbit in July 2004. The two Double Star orbits are aligned with those of ESA's four Cluster satellites, so that all six spacecraft are studying the same region of near-Earth space at the same time (Liu and Escoubet, 2005).

On 7 August 2004, Cluster and TC-1 were in very favorite positions for the study of the temporal and spatial evolution of the plasma sheet activity. In this paper we present simultaneous observations of a reconnection event that occurred between Cluster and TC-1 (the properties of the dipolarizations in this event have been investigated by Nakamura et al. (2005). By using multi-point analysis and the observation of energetic electron and ion flux, we study the movement and structure of current sheet, and discuss the braking effect of an earthward flow burst in the inner magnetosphere. 
Aug. 7, 2004 12:00 to 24:00 UT
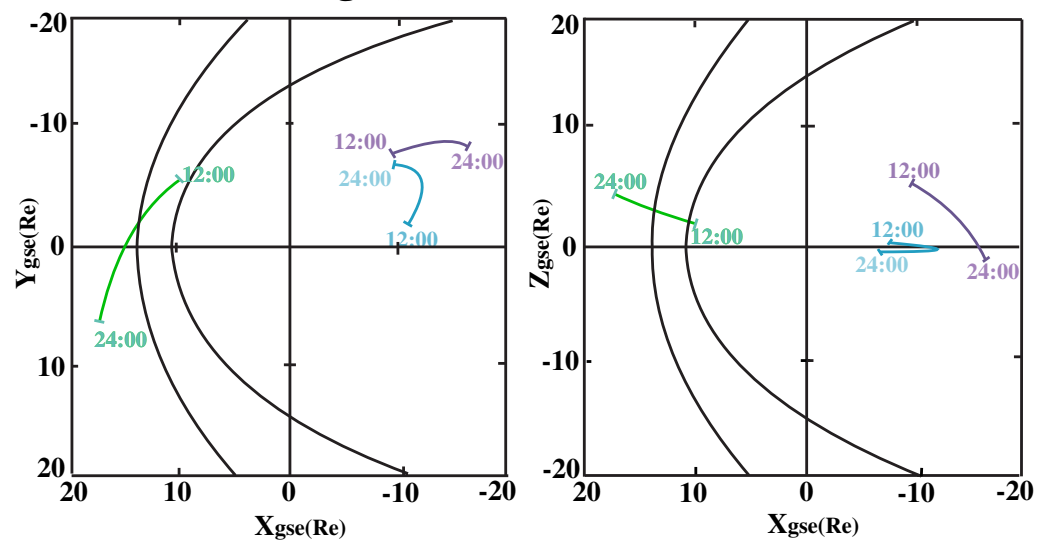

CLUSTER DOUBLESTAR1 GEOTAIL

Fig. 1. The trajectories of Cluster, Double Star and Geotail during the period of 12:00 to 24:00 UT on 7 August 2004.

\section{Observation}

During the period 12:00 UT to 24:00 UT on 7 August 2004, both Cluster, located at about $15 R_{E}$, and TC-1, located at about $10 R_{E}$, were in the post-midnight sector (the trajectories of Cluster, TC-1 and Geotail are displayed in Fig. 1).

Figure 2 show the observation of Cluster and TC-1 during the period of 16:00 to 24:00 UT on 7 August 2004. In the upper part of Fig. 2, from top to bottom, are the magnetic field $B_{x}$ and $B_{z}$ components, and ion flow velocity $V_{x}$ component from Cluster. The bottom part shows the TC-1 data of the magnetic field $B_{x}$ and $B_{z}$ components, and ion flow velocity $V_{x}$ and $V_{y}$ components. All spacecraft data are plotted in the Geomagnetic Solar Magnetospheric (GSM) system. Measurements of the magnetic field and ion distribution function on board Cluster are performed by the fluxgate magnetometer (FGM) experiment (Balogh et al., 2001) and ion spectrometry (CIS) experiment (Rème et al., 2001). The TC-1 data obtained by the fluxgate magnetometer (FGM) experiment (Carr et al., 2005) and Hot Ion Analyzer (HIA) instrument (Rème et al., 2005).

We can see from Fig. 2 that at about 16:33 UT Cluster first observed a tailward flow followed by an earthward flow (panel c), then detected an earthward flow at about 20:45 UT, and finally observed a strong tailward flow followed by an earthward flow at about 22:59 UT. Associated with these plasma flows, magnetic field disturbances are observed and several bipolar signatures in the $B_{z}$ component (panel b) were detected. From panel (f), we can see that beginning at about 16:15 UT, TC-1 observed several earthward flows, then at about 20:30 UT, it detected earthward flows followed by tailward flows, and finally it observed an earthward flow followed by a tailward flow beginning at about 23:06 UT. There are also magnetic field disturbances associated with these plasma flows, and several bipolar signatures in the $B_{z}$ component were observed.
In Fig. 3, the $B_{x}$ magnetic field component, the $\mathrm{X}$ component of the magnetic field curvature vector, and the $V_{x}$ flow velocity component have been displayed in an expanded scale for the period of 23:04 UT to 23:18 UT. We can see that during the flow reversal from the tailward to earthward flow (panel c), the $B_{z}$ changed the sign from mainly negative values to positive values (panel a), and the $\mathrm{X}$ component of the magnetic curvature vector switched sign from mainly a tailward direction to a more earthward direction (panel b), which is consistent with expectations during the passage of a tailward movement X-line. The simultaneous observation of flow reversal from tailward to earthward by Cluster, located at about $15 R_{E}$, and the flow reversal from earthward to tailward by TC-1, located at about $10 R_{E}$, strongly suggests that reconnection occurred between them. By using multi-point analysis, it is found that the propagation of the disturbances of magnetic fields around 23:00 UT was mainly dawnward with a tailward component (Nakamura et al., 2005). This indicates that the initial source of the disturbance is located duskward and earthward of Cluster. Note that Cluster observed the tailward flow beginning at 22:59 UT, MIRACLE/IMAGE magnetograms detected an enhancement in westward electrojet activity starting at 23:00 UT and dispersionless injections were observed by LANL satellites at 23:13 UT (Nakamura et al., 2005).

It is interesting to note that associated with these flows, there are corresponding clear changes in the magnetic field configuration at Cluster and TC-1, i.e., dipolarizations with clear jumps in $B_{z}$, accompanied by a decrease in $B_{x}$. It appears that the dipolarization has proceeded in steps. This means that each flow burst event drives the $B_{z}$ field to a new and higher level of dipolarization, as expected by the magnetic flux pileup model (Shiokawa et al., 1998). The bipolarizations are accompanied by a large amplitude of magnetic field fluctuations, as reported earlier by AMPTE/CCE (Ohtani et al., 1992). Nakamura et al. (2005) have calculated 

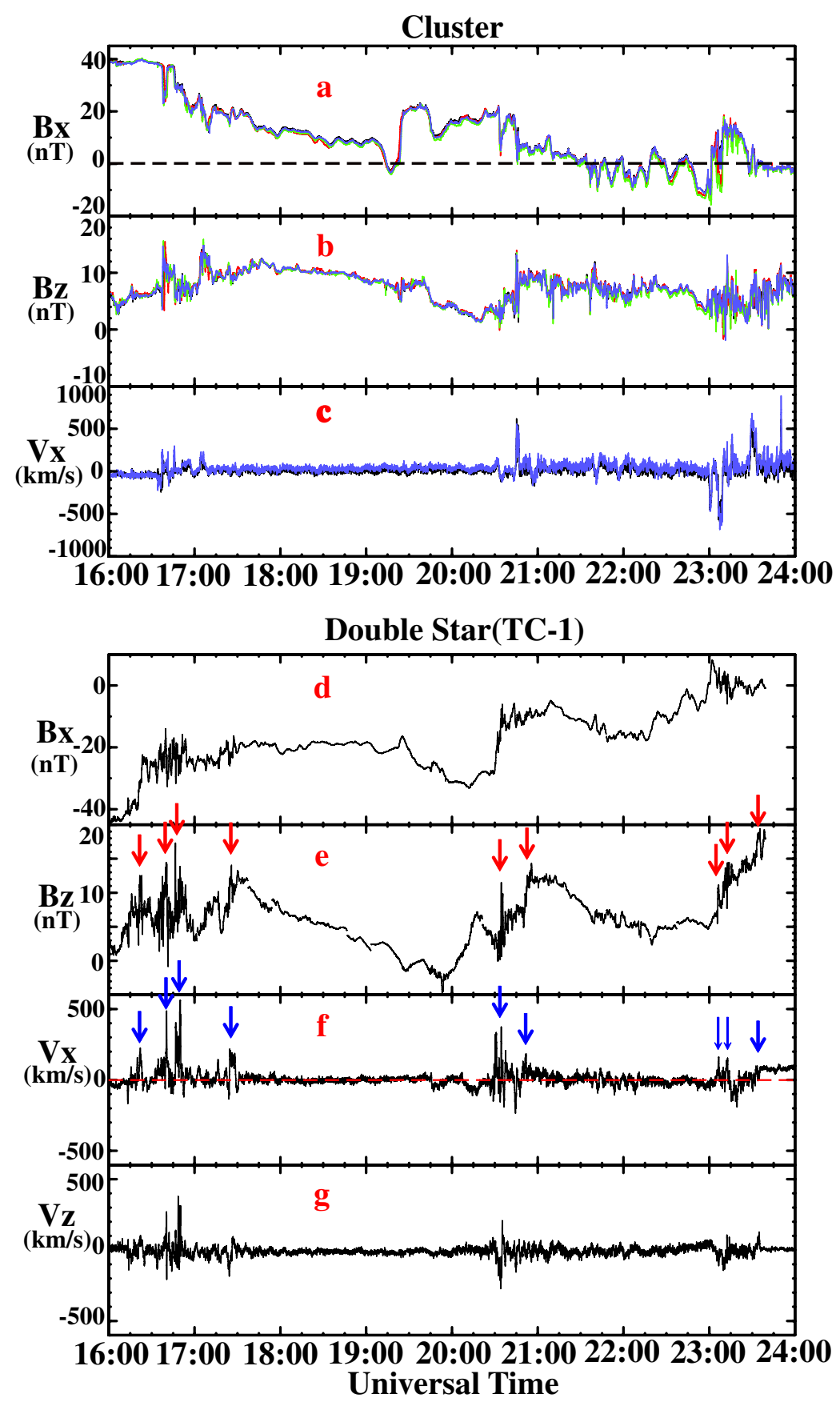

Fig. 2. Overview of the Cluster and Double Star (TC-1) data. In the upper part, from top to bottom, are the magnetic field components of $B_{x}$ and $B_{z}$, and ion flow velocity of $V_{x}$ of Cluster, respectively. At the bottom, from top to bottom, are the magnetic field components of $B_{x}$ and $B_{z}$, and ion flow velocity of $V_{x}$ and $V_{y}$ of TC-1. Vectors are on the GSM frame of reference. SC1, SC2, SC3 and SC4 are represented by black, red, green and blue lines, respectively.

the propagation speed and direction for the $B_{z}$ enhancement around 20:33, 20:44, and 2258 UT in Cluster and compared this with the observation of TC-1. They found that for the first two disturbances, both spacecraft could likely have detected the same disturbances propagated from TC-1 to Cluster. However, the last disturbance cannot be understood in terms of propagation of the same disturbance. This also indicates that the source region is most likely located between
Cluster and $\mathrm{TC} 1$ and the propagated disturbance reached Cluster and TC-1 nearly simultaneously.

As TC-1 was located closer to the Earth, earthward flow bursts are expected to have been decelerated significantly due to the interaction with a strong magnetic field in the inner magnetosphere (Birn et al., 1999). It has also provided an opportunity to study the braking of an earthward flow burst in the inner magnetosphere, to investigate how the 


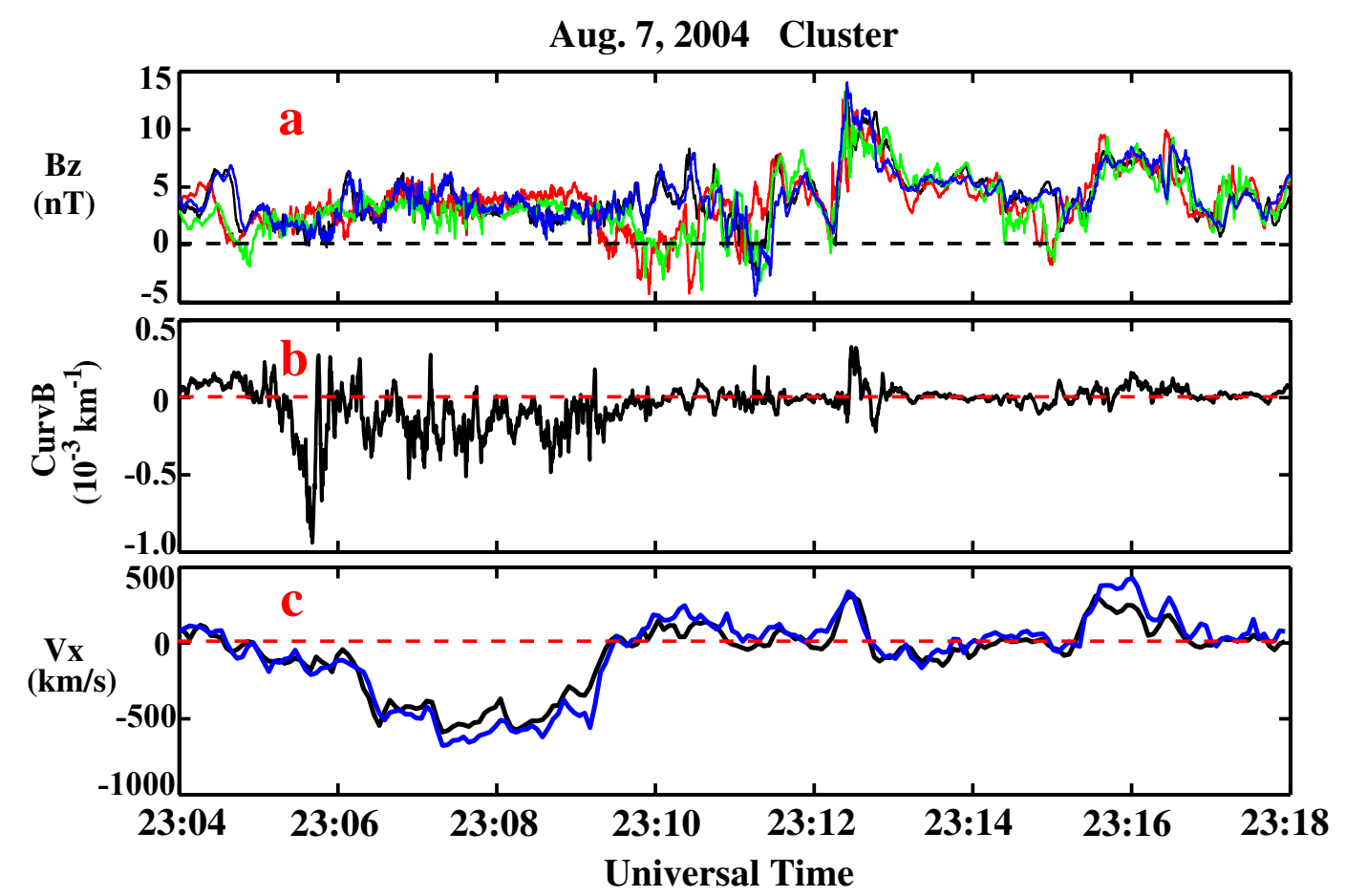

Fig. 3. The magnetic field component of $B_{z}, \mathrm{X}$ component of the magnetic field curvature vector and the flow velocity component of $V_{x}$ observed by Cluster in an expanded time scale during the flow reversal.

interaction of these earthward flow bursts affects the stress balance between the plasma and magnetic fields in the braking region, and the dipolarization of the magnetic field to produce the substorm current wedge (Baumjohann et al., 1999). By comparison of panels (e) and (f) in Fig. 2, we can see that there is a close correlation between the dipolarization indicated by the red arrows and the flow burst indicated by the blue arrows at TC-1 at $10 R_{E}$. We also note that the impulsive earthward flow (panel f) lasts only for several minutes and is followed by "rebounds" where the plasma speed becomes briefly tailward but with a smaller magnitude. Such phenomenon has been observed by Kauristie et al. (2000), Schödel et al. (2001), and Slavin et al. (2002). In addition, we can see from Fig. 2, together with the large earthward flow (panel $f$ ), that there are also large enhancements of the flow in the $\mathrm{Y}$ direction (panel g). This means that the earthward flow burst is companied by a rotation of the flow in the equatorial plane as if the flow was attempting to move laterally around an obstacle.

Figure 4 shows the energetic electron and proton fluxes observed by the RAPID instrument for all four Cluster satellite during the period of 16:00 UT and 24:00 UT on 7 August 2004 (Wilken et al., 1997). When the spacecrafts are located in the lobe, the energy ion and electron intensities are low. While the magnetic field strength drops, indicating that the spacecrafts have moved toward the plasma sheet, the ion and electron intensities begins to rise (Owen et al., 1995). As remote sensing of the edge of the plasma sheet boundary occurs, the increases and decreases of the flux intensity of electrons and ions indicate that the satellites are approaching or moving away from the plasma sheet boundary. The result is nearly consistent with the determination of movement of the current sheet by timing and Minimum Variance Analysis. It is interesting to note that the Cluster fleet crosses the structure in a "first entry and last out" order. Such energetic electrons and ions embedded in an earthward-moving plasma structure have been observed (Zong et al., 2004). The bursts of electrons are well confined in a smaller scale structure than ions, and the four Cluster spacecraft experienced different flux onset timing and profile shapes. As energetic electrons are unique in their capability to fully assess magnetic field line topology, they should be able to clearly delineate the magnetic structure associated with reconnection. More investigation is needed with other observations for detailed study of the small coherent structure.

\section{Conclusion and discussion}

It is a longstanding issue in magnetospheric substorm physics to understand where and exactly when key substorm processes initiate (Baker et al., 2002). One point of view is that very near-Earth $\left(6-8 R_{E}\right)$ instabilities lead to cross-tail current disruption and auroral brightening, and then magnetic reconnection occurs subsequently in the midtail (20$30 R_{E}$ ) region (Lyons, 2000). On the other hand, a case studied in detail using Geotail, geostationary Earth orbit (GEO), and ground-based data, provided considerable evidence that magnetotail reconnection began prior to the near-Earth and 


\section{Aug. 7, 2004 Cluster/RAPID}

(60 sec averages)

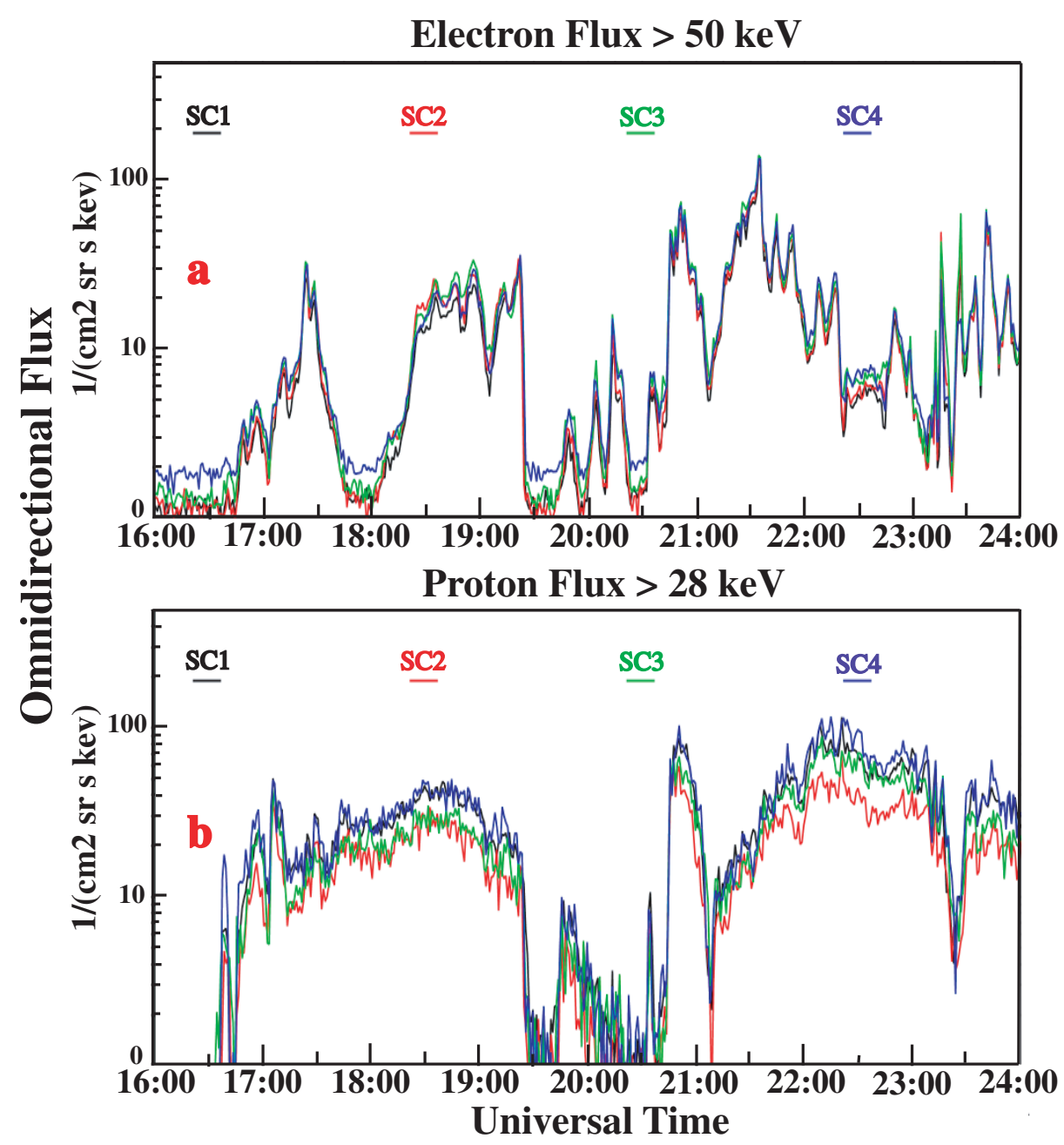

Fig. 4. Energetic electron and proton fluxes observed by RAPID instrument for all four Cluster satellite on 7 August 2004. SC1, SC2, SC3 and SC4 are represented by black, red, green and blue lines, respectively.

auroral onsets of activity (Ohtani et al., 1999). Transient high-speed plasma flows, which are called burst bulk flows (BBF) or flow burst, play a major role in the magnetotail mass, energy and magnetic flux transport in the magnetotail (Baumjohann et al., 1990; Angelopoulos et al., 1996; Nakamura et al., 2004). Possible mechanisms for these flows are patchy impulsive reconnection processes and/or plasma instabilities. How these burst flows at different locations in the tail evolve and interact with the inner magnetosphere is not well understood. It is essential to study the evolution of the flow burst by identifying the temporal and spatial relationship between the flow and magnetic disturbances.

In a good conjunction, Cluster and Double Star spacecraft were able to probe plasma sheet activity near the neutral sheet. In this paper we present observations for one conjugate reconnection event by Cluster and TC- 1 near the neutral sheet on 7 August 2004. The simultaneous observation of a flow reversal at about 22:59 UT from tailward to earthward by Cluster, located at about $15 R_{E}$, and the flow reversal at about 23:06 UT from earthward to tailward by TC-1, located at about $10 R_{E}$, strongly suggests that the reconnection happened near the region between them. Note that Cluster observed a magnetic reconnection event with the tailward flow beginning at 22:59 UT, while MIRACLE/IMAGE magnetograms detected an enhancement in westward electrojet activity starting at 23:00 UT, and dispersionless injections were observed by LANL satellites at 23:13 UT (Nakamura et al., 2005). By using multi-point analysis and observation of energetic electrons and ions flux, we study the propagation of the disturbance, the movement and structure of the current sheet, and discuss the braking effect of the earthward flow burst in the inner magnetosphere. However, from the observations of plasma, magnetic field and flux of energetic electrons and ions, the structure near the reconnection region 
is very complex. It is possible that many small-scale reconnection tearing vortices coalesce into a coherent structure, such as a plasmoids (Slavin et al., 2003; Deng et al., 2004; Hoshino et al., 1994; Sergeev et al., 1996; Shay et al., 2003), by multiple $\mathrm{X}$-line reconnection.

Acknowledgements. We thank all members of the Cluster and Double Star teams for the high quality data and for the successful spacecraft operation. X. H. Deng appreciates National Changjiang Scholarship Project at Wuhan University, the support of Outstanding Young Scientist Founding of China (40325012), National Science Foundation of China (40390151), Funding for the Doctoral Program of Higher Education, and the support of ÖAD for a visiting professorship at the Space Research Institute, Graz, Austria.

Topical Editor T. Pulkkinen thanks two referees for their help in evaluating this paper.

\section{References}

Angelopoulos, V., Coroniti, F. V., Kennel, C. F., et al.: Multipoint analysis of a bursty bulk flow event on April 11, 1985, J. Geophys. Res., 101, 4967-4989, 1996.

Baker, D. N., Pulkkinen, T. I., Angelopoulos, V., Baumjohann, W., and McPherron, R. L.: The neutral line model of substorms: Past results and present view. J. Geophys. Res., 101, 12 975-13010, 1996.

Baker, D. N., Peterson, W. K., Eriksson, S., et al.: Timing of magnetic reconnection initiation during a global magnetospheric substorm onset, Geophys. Res. Lett., 29(24), 2190, doi:10.1029/2002GL015539, 2002.

Balogh, A., Carr, C. M., Acuña, M. H., et al.: The Cluster magnetic field investigation: overview of in-flight performance and initial results, Ann. Geophys., 19, 1207-1217, 2001,

\section{SRef-ID: 1432-0576/ag/2001-19-1207.}

Baumjohann, W., Paschmann, G., and Lühr, H.: Characteristics of high-speed ion flows in the plasma sheet. J. Geophys. Res., 95, 3801-3810, 1990.

Baumjohann, W., Hesse, M., Kokubun, S., Mukai, T., Nagai, T., and Petrukovich, A. A.: Substorm Dipolarization and recovery, J. Geophys. Res., 104, 24 995-25 000, 1999.

Birn, J., Hesse, M., Hardened, G., Buamjohann, W., and Shiokawa, K.: Flow, braking and the substorm current wedge, J. Geopys. Res., 104, 19 895-19904, 1999.

Carr, C. M., Brown, P., Zhang, T. L., et al.: The Double Star magnetic field investigation: instument design, performance and highlights of the first year's observation, Ann. Geophys., 23, 2713-2732, 2005.

Deng, X. H., Matsumoto, H., Kojima, H., Mukai, T., Anderson, R. R., Baumjohann, W., and Nakamura, R.: GEOTAIL encounter with reconnection diffusion region in the Earth's magnetotail: evidence of multiple $\mathrm{x}$-lines collisionless reconnection?, J. Geophys. Res., Vol. 109, No. A5 A05206, doi:10.1029/2003JA010031, 2004.

Hoshino, M., Nishida, A., Yamamoto, T., and Kokubun, S.: Turbulent magnetic field in the distant magnetotail: Bottom-up process of plasmoid formation?, Geophys. Res. Lett., 21, 2935-2938, doi:10.1029/94GL02094, 1994.

Kauristie, K., Sergeev, V. A., Kubyshkina, M., et al.: Ionospheric current signatures of transient plasma sheet flows, J. Geophys. Res., 105, 10 677-10 690, doi:10.1029/91JA01832, 2000.
Liu, Z. X., Escoubet, C. P., Pu, Z., et al.: The Double Star mission, Ann. Geophys., 23, 2707-2712, 2005.

Lui, A. T. Y., Chang, C. L., Mankofsky, A. Wong, H. K., and Winske, D.: A cross-field current instability fro substorm expansions, J. Geophys. Res., 96, 11389-11401, doi:10.1029/91JA00892, 1991.

Lyons, L. R.: Determinations of relative timing of near-Earth substorm onset and tail reconnection, ESA SP-443, 255, 2000.

Nakamura, R., Baumjohann, W., Mouikis, C., et al.: Spatial scale of high-speed flows in the plasma sheet observed by Cluster, Geophys. Res. Lett., 31, L09804, doi:10.1029/2004GL019558, 2004.

Nakamura, R., Baumjohann, W., Zhang, T. L., et al.: Cluster and Double Star observations of dipolarization, Ann. Geophys., 23, 2915-2920, 2005.

Ohtani, S., Takahashi, K., Zanetti, L. J., Potemra, T. A., et al.: Initial signatures of magnetic field and energetic particle fluxes at tail reconfiguration: Explosive growth phase, J. Geophys. Res., 97, 19311-19324, doi:10.1029/92JA01832, 1992.

Ohtani, S., Creutzberg, F., Mukai, T., Singer, H., Lui, A. T. Y., Nakamura, M., Prikryl, P., Yumoto, K., and Rostoker, G.: Substorm onset timing: The December 31, 1995 event, J. Geophys. Res., 104, 22 713-22 728, doi:10.1029/1999JA900209, 1999.

Owen, C. J., Slavin, J. A., Richardson, I. G., Murphy, N., and Hynds, R. J.: Average motion, structure, and orientation of the distant magnetotail determined from remote sensing of the edge of the plasma sheet boundary layer with $\mathrm{E}>35 \mathrm{keV}$ ions, J. Geophys. Res. , Vol. 100 , No. A1, 185-204, 1995.

Rème, H., Aoustin, C., Bosqued, J. M., et al.: First multispacecraft ion measurements in and near the Earth's magnetosphere with the identical Cluster ion spectrometry (CIS) experiment, Ann. Geophys., 19, 1303-1354, 2001,

SRef-ID: 1432-0576/ag/2001-19-1303.

Rème, H., Dandouras, I., Aoustin, C., Bosqued, J. M., Sauvaud, J. A., Cao, J. B., Shi, J., Pu, Z., Bavassano-Cattaneo, M. B., Parks, G. K., Carlson, C. W., Klecker, B., Moebius, E., Kistler, L., Korth, A., Lundin, R., Escoubet P., and the HIA team: The HIA instrument on the Tan Ce 1 Double Star near-equatorial spacecraft and its first results, Ann. Geophys., 23, 2757-2774, 2005.

Schödel, R., Baumjohann, W., Nakamura, R., Sergeev, V. A., and Mukai, T.: Rapid flux transport in the central plasma sheet, J. Geophys. Res., 106, A1, 301-313, 2001.

Shiokawa, K., Baumjohann, W., Haerendel, G., et al.: Highspeed ion flow substorm current wedge formation and multiple Pi2 pulsations, J. Geophys. Res., 103, 4491-4508, doi:10.1029/97JA01680, 1998.

Sergeev, V. A., Angelopoulos, V., Gosling, J. T., Cattell, C. A., and Russell, C. T.: Detection of localized, plasma deleted flux tubes or bubbles in the midtail plasma sheet, J. Geophys. Res., 101, 10 817-10 826, doi:10.1029/96JA00460, 1996.

Shay M. A., Drake, J. F., Swisdak, M., Dorland, W., and Rogers, B. N.: Inherently three dimensional magnetic reconnection: A mechanism for burst bulk flows?, Geophys. Res. Lett., 30, 1145, doi:10.1029/2002GL016267, 2003.

Slavin, J. A., Fairfield, D. H., Lepping, R. P., et al.: Simultaneous observations of earthward flow bursts and plasmoid ejection during magnetospheric substorms, J. Geophys. Res., 107(A7), 1106, doi:10.1029/2000JA003501, 2002.

Slavin J. A., Lepping, R. P., Gjerloev, J., Fairfield, D. H., et al.: Geotail observations of magnetic flux ropes in the plasma sheet, J. Geophys. Res., 108(A1), 1015, doi:10.1029/2002JA009557, 2003. 
Wilken, B., Axford, W. I., Dsglis, I., Daly, P., et al.: Rapid: the imaging energetic particle spectrometer on Cluster, Space Sci. Rev., 79, 399-473, 1997.
Zong Q. G., Fritz, T. A., Pu, Z. Y., Fu, S. Y., et al.: Cluster observations of earthward flowing plasmoid in the tail, Geophys. Res. Lett., 31, L18803, doi:10.1029/2004GL020692, 2004. 